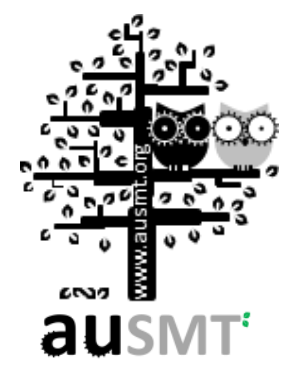

\title{
Analysis of dynamic path loss based on the RSSI model for rupture location analysis in underground wireless sensor networks and its implications for Earthquake Early Warning System (EEWS)
}

\author{
P. K. Dutta1, ${ }^{\text {, }}$, O. P. Mishra ${ }^{2}$, and M. K. Naskar ${ }^{3}$ \\ ${ }^{1}$ Research Fellow, Advanced Digital Embedded System Lab, Electronics and Telecomm. Dept., Jadavpur University, Kolkata, India and \\ Faculty, Electronics \& Automation, D.G. Shipping, India \\ ${ }^{2}$ Scientist -,National Centre of Seismology, Ministry of Earth Sciences, India \\ ${ }^{3}$ Advanced Digital Embedded System Lab, Electronics and Telecomm. Dept., Jadavpur University, India \\ (Received 12 January 2015; Accepted 25 February 2015; Published on line 1 September 2015) \\ *Corresponding author email: ascendent1@gmail.com \\ DOI: 10.5875 /ausmt.v5i3.858
}

Abstract: Sensors deployed in underground tunnels found that radio frequency signals suffer significant signal strength attenuation which can result in considerable variation of link quality on the receiving end. This study analyzes the received signal strength index (RSSI) based on the development of a theoretical wireless sensor model for data collection by enabling sensors to determine the location from which each data packet is obtained. To improve positioning accuracy, the complex radio wave propagation environment requires the use of a voronoi cell to minimize signal attenuation. A relatively simple calculation is used to predict the intensity and perception range of the received wireless signals to measure the extent of signal reduction in the attenuating rock medium. Simulation results show that RSSI-based localization and wireless network lifetime and throughput measurements are more accurate when the node concept is applied to the self-locating rupture zones than the maximum likelihood estimation method. The proposed minimum energy relay routing technique based on beacon node chain deployment is found to help correct localization errors resulting from interference caused by the underground tunnel environment. The extent of localization and power of the sensor nodes are determined based on the beacon node chain deployment of tunnel wireless sensor networks. The algorithm accounts for the distance and the corresponding RSSI between adjacent beacon nodes to calculate the actual path loss parameter in the tunnel. The proposed model can serve as the theoretical basis for locating ruptures in underground wireless sensor network nodes, thus maximizing the monitoring range of large scale tectonic environments while minimizing equipment cost. We recommend that this model can be field tested through a series of experiments by researchers and engineers working in seismology, telecommunication, and information technology.

Keywords: Underground node position, chain deployment, received signal strength indicator (RSSI), path loss parameter

\section{Introduction}

Earthquakes are one of the most devastating natural geospatial hazards in areas of frequent seismic activity extending from inland areas to coastal belts due to sub- oceanic earthquakes associated with subduction dynamics [1 - 2]. The most significant countermeasures to mitigate earthquake damage focus on automatic and manually forecasting systems to provide early warnings [3 - 5]. However, producing accurate warnings is highly challenging as it involves path communication through 
the multiple layers of rock, which acts as an attenuating medium. In the proposed study, transmission characteristics of wireless sensors deployed in the rock medium are found to have been affected due to the attenuation associated with the received signal strength and result in significant differences in the link quality at the receiving end which affect the identification of the exact positioning of wireless sensing, thus maximumizing monitoring range while minimizing cost. Work on an Earthquake Early Warning Module began in the previous decade, and it took several years for researchers to understand the intricate mechanisms needed to generate precise information regarding the diagnostic parameters that determine earthquake events. The different dynamic stages of a seismic evolution process is found to effect the the design and functioning stages associated with earthquake early warning systems because of the complexity involved in the physics of earthquake genesis [4]. The particular spatial and temporal characteristics of regional earthquake activity can be monitored using instruments with sensors/transducers which can retrieve data and transmit information over electronic circuitry. However, seismic monitoring is fundamentally limited by

P.K.Dutta has worked under the guidance of Dr Mrinal Kanti Naskar and Dr O. P. Mishra in studying the effect of interdisciplinary studies in catastrophic analysis and risk mechanism in Advanced Digital and Embedded System Laboratory, Jadavpur University. The major focus of research is the study of complex processes involved in Earthquake Genesis Mechanism Validation and Warning System Design. He published about 20 peer reviewed journals in the past 3 years. He has expertise on the earthquake generating mechanism and forecast system, computational intelligence, operating system, signal processing and wireless communication. He has done this work by using realtime acceleration data recorded by various institutions of India available freely from India Meteorological Department (IMD), New Delhi after the Sikkim Earthquake of 2011.

O.P. Mishra has degree of Doctor of Science (D.Sc.) from Geodynamics Research Center (GRC), Ehime University, Japan for his outstanding research in the field of seismic tomography. His research has unraveled several intricate seismo-tectonic settings beneath the sub-oceanic regions of Japan, Pacific and India. He is an expert of Applied Geophysics and Solid Earth Science dealing with seismological research and disaster risk management studies on earthquake generating mechanism, earthquake precursor in diverse geotectonic environ of the world. He authored more than 100 peer reviewed papers and reports in national and international journals of repute. He contributed significantly in the field of geosciences and disaster management by addressing several pertinent issues of disaster management science of the SAARC region of 8- member states (Afghanistan, Bangladesh, Bhutan, India, Maldives, Nepal, Pakistan, Sri Lanka) during his tenure at SAARC Disaster Management Centre (SDMC), New Delhi as its Head / Director. Currently, he is working with the Ministry of Earth Sciences, India as one of the senior level scientists of India. He is the recipient of National Mineral Award- 2008 by the Government of India and A. S. Arya Disaster Prevention Award-2014 by IIT Roorkee, India in the field of Disaster Management.

M .K Naskar received his B.Tech (Hons.) and M.Tech from E\&ECE Dept., IIT Kharagpur in 1987 and 1989 respectively and Ph.D. from Jadavpur University in 2006. He served as a faculty member in NIT, Jamshedpur (then RIT Jamshedpur) and NIT, Durgapur (REC Durgapur) from 1991-1996 and 1996-1999 respectively. Currently he is a Professor in the Department of Electronics and Telecommunications Engineering, Jadavpur University, Kolkata, India and incharge of the "Advanced Digital and Embedded Systems Lab". His research interests include Wireless Sensor Networks, Optical Networks and Embedded Systems. signal attenuation associated with the identification of the triggering mechanisms associated with rock bursts or the asperity zone and the inability to recover all useful information from waveforms [6]. The measured earthquake records constitute only a small part of continuous record after a seismic rupture initiation that can influence the seismic triggered event analysis, involving a pre-fixed time interval of a continuous record of time-series data for pre and post event analysis. Conventional models for interpreting the physical state of the crust before and after earthquakes often fail to explain the associated fault dynamics and stress transfer mechanism involved in seismogenesis [6-7]. The major constraint in the development of scientific instrumentation for use in an earthquake early warning system (EEWS) is related to the uncertainty related to the ground truth location and inaccuracy arising from errors in location and focal depth. The second major constraint for the development of a feasible EEWS is the latency incurred in delivering accurate locational data to first responders. Results from integrated geophysical surveys can be interpreted to provide insight into the distribution of physical rock properties [4], and rock analysis can be localized by analyzing continuously recorded physical fields and wave propagation to provide advance warning of impending calamity. An accurate location analysis of the seismic rupture can allow researchers to infer whether a slow or weak rupture starts at a certain point, with the closest site(s) recording waves radiating from that very point that results in the formation of a nucleating patch, whilst others may only record waves generated later in the rupture process by a higher stress drop at the patch of the same source. Detailed regional geophysical observations allow researchers to create an image providing insight into fault lock locations, and point to particular locked patches with the clearest potential for future large-scale earthquakes. Limits to current technology and scientific understanding regarding the processes controlling fault locking and release prevent scientists from precisely predicting the location and timing of future earthquakes. Spatio-temporal gradients of seismic parameters and other patterns are judged by their location and timing through analysis of the asperity localization is a problem from the point of view of stress waves.

Wireless communication is regarded as one of the fastest tools to communicate diagnostic information about rupture locations, thus allowing data to be transmitted to responders well before the earthquake occurs [8]. A useful EEWS has to provide real-time event estimates to remote sensing sites before the strong shaking (S-wave) arrives. However, wireless communication through rock mass is complicated by the 
significant path loss involved in the process. Most of the current research on wireless signal attenuation focused on specific environments and very few studies have addressed wireless signal transmission over gradually changing environments. Through this study, we can predict the coverage range resulting from the local attenuation of ground motion and site effects for location error analysis and computing the probability of exceeding a given level of ground motion for different time intervals [9]. The measurement structure of the estimation principle of the RSSI attenuation model is closely related to the location site of the antenna which is reflected through non-linear equations [10]. Specialized WSN protocols are needed to fully realize autonomous geophysical monitoring procedures using the selfpotential monitoring technique. Specially, in-network localization protocols that produce accurate node location estimates are required to enable autonomous operation. Energy is the most important criterion for achieving continuous remote monitoring through optimizing transmission to determine the remaining energy at each node, which is then used to produce a statistical analysis of energy consumption with a significant set of nodes and to then extrapolate the results to the whole system via an analog to digital converter (ADC) which can determine the node's remaining energy. The present study analyzes the dynamic path based on the received signal strength index to accurately identify rupture locations for a given underground wireless sensor network setup.

\section{Spatial Distribution of Sensors}

The location of a seismic event is assumed to be a point within the seismic source that triggers a set of seismic sites. Accurate location determination depends on the nature of the rupture process at the source. Specific arrival times are needed to determine the location of rupture initiation, otherwise the location will be a statistical average of different parts of the same source. In recent years, work on the localization problem in Underwater Wireless Sensor Networks has produced many innovative solutions and ideas [9]. One of its key points is the noise level of the acquisition system. Subsequently seismological processing (e.g., seismic source parameter and attenuation or velocity inversion) depends on the locations of sensors/recorders. All subsequent interpretation of individual events depends on the location of such devices, and it is important to address events at a distance from active mining areas, close to a shaft or in places generally not predicted by numerical modeling as likely sites for seismic events. Localization around planes, migration, spatial-temporal gradients of seismic parameters and other patterns are judged by their location and timing. We investigate various propagation models and fading techniques to achieve a better understanding of the rupture locations for wireless sensor networks. The strength of the radio signal is recorded using the radio signal strength indicator (RSSI) appended to each packet header received by the transceiver. To understand how radio signal strength varies as a function of range, the test is repeated with the wireless sensor placed at varying distances from the PC server. RSSI was used for location error analysis. The true location can be determined using ambient noise, but focal depth analysis does not provide accurate location information. To transmit data efficiently over wireless channels in WUSNs, existing schemes set minimum transmission levels to maintain signal reliability as determined by received signal strength at the antenna. In this topology, nodes are placed in a linear architecture and the base station is placed far from the sensor node. A single-hop transmission can be used for linking data. To access the base station, a sensor node receiver designed as in (Fig. 1) has an oscillatory circuit which is set to the appropriate frequency. If the signal, which is transmitted from the base station, corresponds to a receiver frequency, the modulated signal goes from the receiver to the electronic switch 'SW', which turns on the output cascade of the transmitter ' $T x^{\prime}$ '.

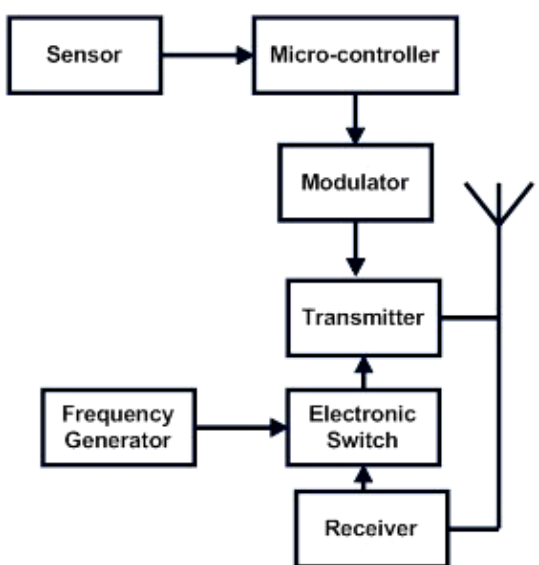

Figure 1. Sensor node block diagram (modified after Nikolaevna and Konstantin [11])

The sensor, the microcontroller, frequency generator and modulator work constantly to achieve effective transmission of anomaly data from the mobile station to the base station. The highly heterogeneous makeup of the underground environment (including soil, rock, coal, etc), is significantly more challenging for wireless communication than air. A collective monitoring scheme for earthquake early warning system requires Rapid Response (Dial-up), Early Warning (On-line) and Structural Monitoring (Off-line) stations, which essentially use an identical instrumentation package consisting of a sensor (Transducer), a digitizer/recorder, an RS-232 remote interrogation communication module and a Timing Module (GPS) with a power supply and external 
charger (220V). The base station (Fig. 2) consists of a microcontroller (which can be connected to an output port using a USB interface), and a frequency generator (Fig. 3) comprising a modulator, transmitter, receiver and antenna.

Base station microcontroller (Fig. 2) submits the frequency generator controlling voltage which corresponds to that of one of the sensor nodes. As a result, the varicap changes capacity, thus changing the generated frequency.

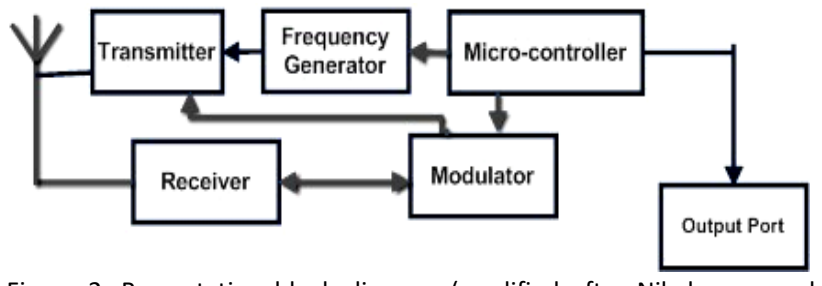

Figure 2. Base station block diagram (modified after Nikolaevna and Konstantin [11])

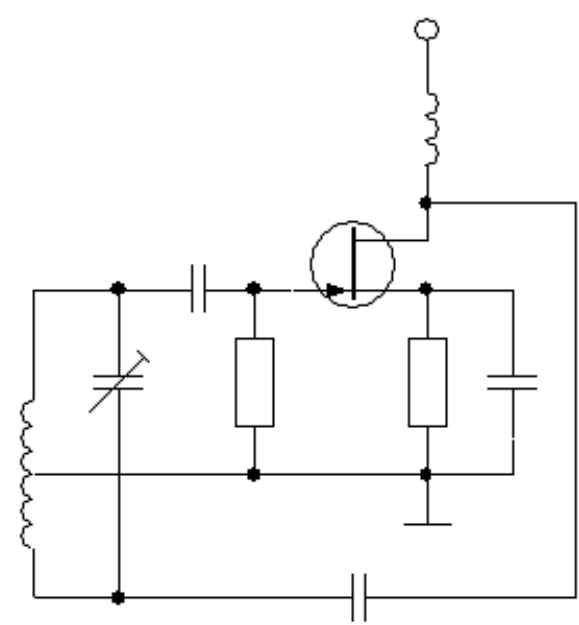

Figure 3. Sensor node frequency generator (modified after Nikolaevna and Konstantin [11]).

The basic circuit is designed to pick up RF signals generated at the sensor frequency generator (Fig. 3) and generate an output equivalent to the signal strength at the base station as shown in (Fig. 4). To implement a power control link, the remote device must implement a control output voltage to trigger an alarm at the corresponding node. This can be done only by using a costly seismosensor for a certain precursor to provide real-time emergency earthquake information to the connected sink nodes in an area measuring a few square-kilometers. The emergency detection nodes start broadcasting earthquake information and form a robust mesh network [12] as described in the WSN-based disaster prevention control system diagram. Using RSSI data we found that, although the total radiated energy cannot be measured, the average strain energy that causes a rupture is a very important for determining the relationship between seismic moment and magnitude, and the relationship between strain energy and seismic moment can be used to identify the depth varying rupture properties. The base station receives the signal from the corresponding sensor node and changes transmitting frequency to check the next node. This cycle continues until all sensor nodes are checked. When the power of the signal received from sensor node is determined to be insufficient, the base station submits an alarm signal to initiate diagnosis of the particular sensor node. Sensor node checking is performed after defined time intervals as each of the scattered sensor nodes can collect and forward data back to the target sink node connected with the power control device (PCD) and other monitoring systems.

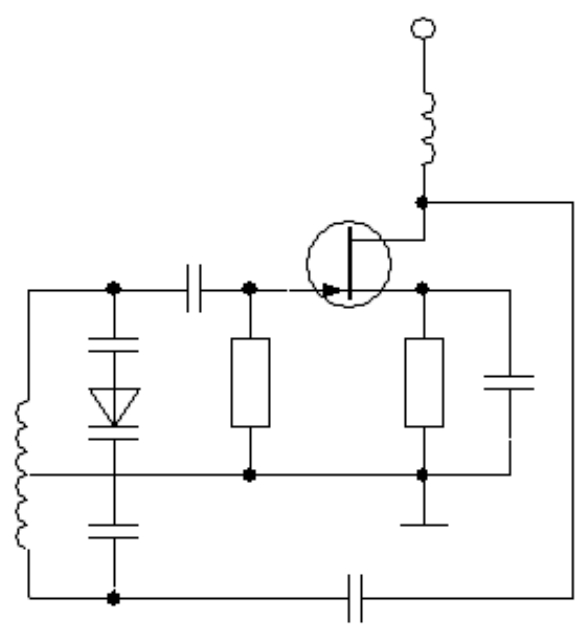

Figure 4. Base station frequency generator (modified after Nikolaevna and Konstantin [11]).

Achieving accurate location estimates based on RSSI requires the network path-loss model which can be accomplished using channel modeling prior to system deployment. However this is unrealistic in dynamic environments like earthquake monitoring scenarios as underground earthquake nodes may become inactive. Secondly, RSSI-based location determination can be easily affected by multipath interference. The time span over which such analyses are done depends on the nature of the rock mass behavior and the scale of analysis. Location interpretation accuracy depends on the nature of the rupture process at the source. Given a slow or weak rupture starting at a certain point, the closest site(s) may record waves radiated from that very point while others may only record waves generated later in the rupture process by a higher stress drop for the zone associated with the same source. Locating the rupture source requires specific information based on the determination of arrival times, otherwise the location will be a statistical average of different parts of the same source. The directional antenna concentrates the RF energy into a specific beam direction for higher RSSI measurements along with greater communication ranges. Location error depends on data accuracy. Positioning methods can be divided into two kinds of positioning algorithms, based on 
the algorithm's scope and distance. The range-based algorithm uses the maximum likelihood method or trilateral rule to measure the distance between nodes to calculate the location of an unknown node. Certain ranging methods including time of arrival [13] and time difference of arrival [14] have been widely used to measure distance between nodes based on the RSSI (Received Signal Strength) consumed by the nodes. Various solutions have been proposed based on two ranging techniques, specifically path loss models based on radio RSSI [15] or acoustic signal strength [16] for identifying signal performance parameters based on signal attenuation and intensity variance with direct line of sight related to the signal propagation distance.

\section{RSSI Attenuation Model for Gradually Changing Environments}

Wireless signal attenuation is closely related to RSSI. The reception of each data frame produces a corresponding RSSI value which reflects the current signal attenuation situation. We can measure the RSSI signal strength of beacon node consumption to determine the distance between nodes. RSSI is a low-cost alternate for long or short-distance measurements in WSN networks, as dynamic instabilities of small scales (micro-seismicity) are induced and activated during the nucleation process. The wireless signal transmission characteristics in gradually changing environments are affected by the redundancy associated with the positioning of beacon nodes that cannot model the effect of the RSSI values, along with the positioning of the unknown nodes to determine an effective signal transmission range for the transmission of each message and the transmission power level. Beacon node selection is performed based on the unknown node which selects the beacon node with the greatest RSSI value and then calculates the position of the unknown node. In [17], the authors described a new range-free localization technique using a mobile beacon for underwater acoustic sensor networks. The mobile beacon periodically broadcasts a beacon message containing its location. To estimate the location, the system obtains a set of potential location candidates using the bi-lateration method and then localizes the sensor node through the weighted mean of all the potential locations with weights computed based on residuals. This improves the localization accuracy and is more tolerant to errors in estimating the distance between the projected beacon points and sensor nodes. Accurate location is a prerequisite for any meaningful study of the mechanics of incipient earthquake behavior. The location error of the proposed early warning location method is composed of two parts. The error in the first part is caused by a lack of information, that is, the information from the first three triggered stations is not sufficient to obtain the an accurate epicenter position that can only be restricted to a segment of a straight line with a limited length, which is deemed an absolute error. The error in the second part is caused by the calculation formula, that is, errors of each parameter in the formulas as shown in [18] inevitably induced by the influence of seismic phase pick-up. This inevitably produces an error in the location result, which is deemed a relative error. To reflect the quantitative stability and reliability of the location method, the relative error is assumed to be caused by the location equation for focal distance of the first triggered station. A routing protocol which can meet the requirements of underground wireless sensor networks has a decisive role on network monitoring quality and survival time. By combining the beacon node chain deployment of tunnelbased Wireless Sensor Networks, a Minimum Energy Relay Routing (DMERR) technique based on the dynamic path loss parameter is implemented to save communication energy to obtain the exact path loss parameter after attenuation. Location analysis based on detailed surface geology data would give a fair idea about ground behavior during an earthquake. The location determination also depends on the numerical procedure used to solve the system of nonlinear site equations. Denser station networks and more accurate data have a reduced impact on the numerical procedure, and allow the algorithm to yield factual estimates of the locationbased parameters. The complexity of the source of a seismic event is described not only by its time, location, radiated seismic energy, seismic moment, and size, but seismicity is also quantified by parameters related to changes in the stress, strain and rheology of the seismic deformation processes. This type of quantification would allow the for integration of seismic monitoring results with numerical rheological models that capture the underlying dynamics. The authors in [19] proposed an RSSI-based cooperative anomaly detection scheme for wireless sensor networks. They were mainly concerned about maintaining perfect sensor nodes positioning. The present study uses RSSI data collected from the received packets. RSSI range-based schemes rely on range of transmission [20] and bearing [21] to determine target or sensor node locations, which generally requires more computations but yields more accurate estimates [21 22]. This procedure requires an accurately-located master event (e.g., a blast) in the proximity of the event to be located, with reliable arrival times at sites used in the relocation procedure. It is assumed here that the velocities of the seismic waves from the master event to the sites and those from the target event are the same. Most previous studies have found that the time period 
over which the strain change associated with the rock can affect the seismic relaxation time [22]. For the stressrelated parameter associated with the structural heterogeneities, a shorter period is required since the cumulative strain over a period of time is to be compared with the present state of stress beneath the sub-surface rock mass affected by the presence of cracks and fractures under complex geotectonic settings for different regions [23 - 26].

\section{Using Wireless sensors for Earthquake Early Warning: Challenges and Constraints}

The EEWS might only issue an alarm if a certain number of stations have triggered. As earthquake early warning systems have only a very short response time, it is necessary to continuously locate nodes so that underground embedded sensors can transmit information to the surface station while minimizing path effects.

It is useful to know the distribution of actual recurrence times about the estimated mean. A key issue in this method of transmission is the problem associated with network efficiency for underground communication between the buried sensors at different depths for network management and data relay systems. The data in the network is collected through underground-to- aboveground communication. Although the deployment of aboveground sensor nodes is quite easy, underground nodes suffer from significantly increased energy requirements and reduced network lifetimes. Each sensor node is assigned a distinctive identifier (ID). The sensor transmitter dissipates more energy than the receiver as it requires more energy for amplification. The base station is located far away from the sensing field to reduce signal attenuation, effectively extend the transmission distance, increase coverage, control equipment costs and maximize the monitoring range through an optimal perception node that remains stationary after deployment. A gateway node is deployed in the same network field at the center of the network. The gateway node is stationary after deployment and rechargeable (Figs. 3-4). Given the importance of the nodes' transmission capability, it is difficult to use homogeneous sensor nodes with identical computational and sensing capabilities in the remote monitoring of seismic activity. To optimize network performance, logical regions are extracted based on the node location in the network in relation to the Base Station (BS). Nodes in first region use direct communication and transmit their data directly to the BS. Similarly, nodes near the gateway form region two and send their data directly to the gateway which aggregates and forwards the data to the BS. Nodes are considered dead after consuming 0.5 joules. On the other hand, in $\mathrm{LEACH}$, nodes die quickly as the network stability period

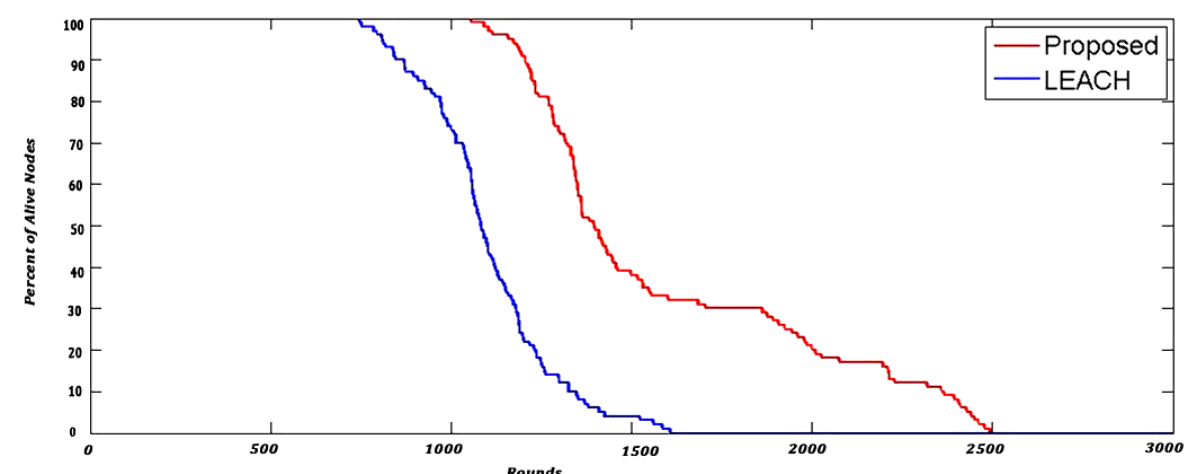

(a)

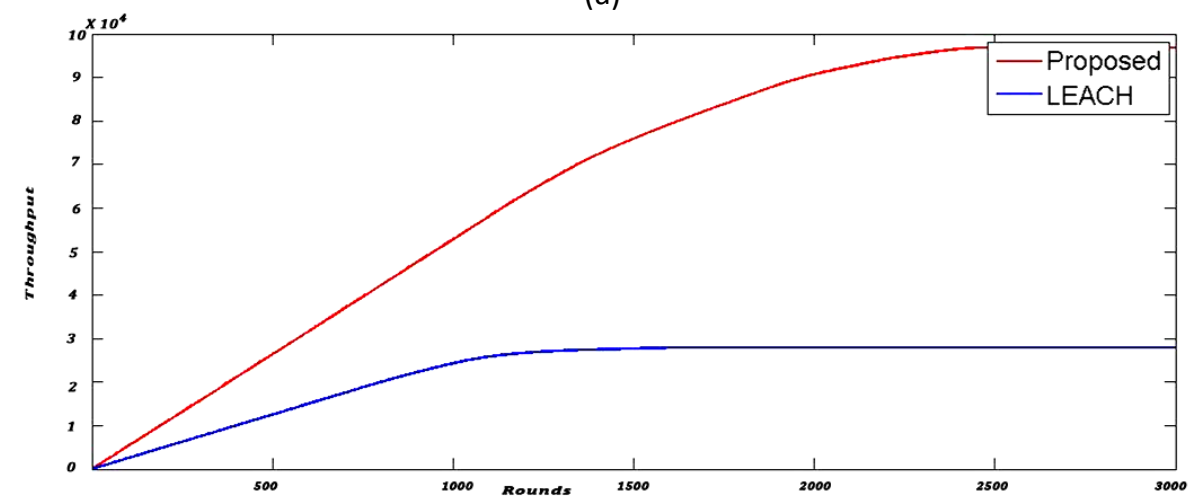

(b)

Figure 5.(a) Interval plot- Analysis of network lifetime following the approach in Dutta et al., 2013 [27].(b) Interval plot- Analysis of Throughput following the approach in Dutta et al., 2013 [27]. 


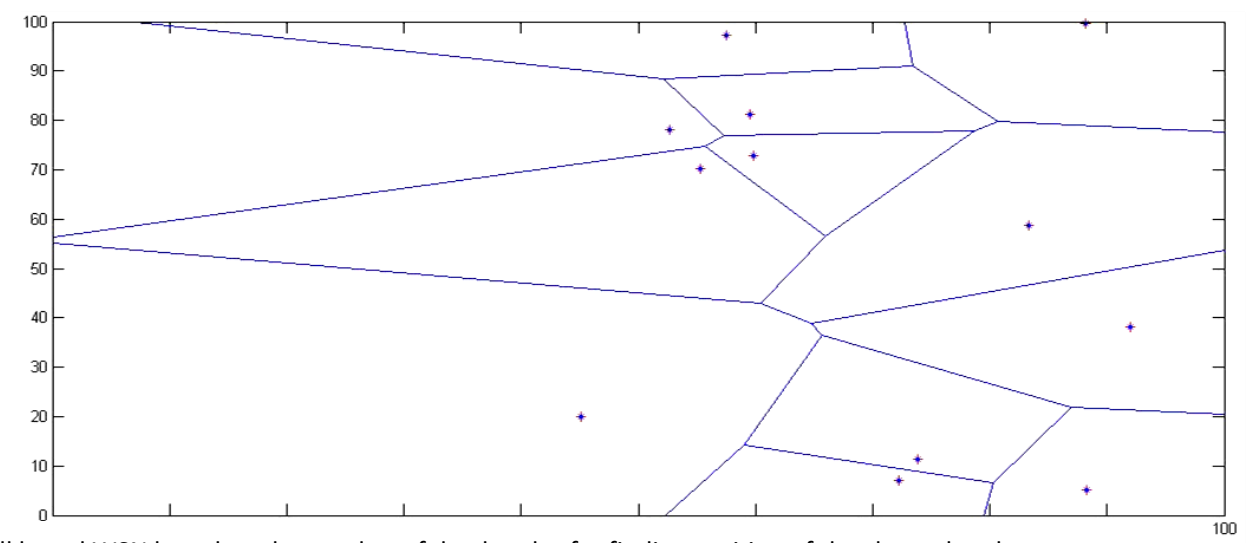

Figure 6. Voronoi cell based WSN based on the number of dead nodes for finding position of the cluster heads.

ends. The algorithm performs better in identifying nonclustered regions and performs efficiently in terms of network lifetime and throughput as shown in Fig. 5(a-b) based on [27]. It is not evident that predestined clusterheads (CHs) in LEACH are distributed uniformly throughout the network field in the M-Gear protocol. Nodes in non-clustered regions use direct communication and transmit their data directly to the BS as the distance between these nodes and the BS is very short. A voronoi cell can give accurate location information based on seismic noise and depth. Parameters involving transmission and residual energy, energy consumption rate, queue size, centrality of cluster head allocation and proximity to the base station provide key information that can be used to select a suitable cluster head based on the spatial characterization. Selection of a favorable clusterhead in earlier rounds result in an inhomogeneous clusterhead routing in later rounds since LEACH tries to distribute energy consumption among all nodes. To obtain the network among sensor nodes, we have re-evaluated and edited the M-GEAR protocol, which is a Gateway-Based Energy-Aware Multi-Hop Routing Protocol designed by [28]. Therefore, there is a possibility that the selected CHs will be concentrated in one region of the network lifetime to balance energy consumption.

Hence, some nodes will not have any $\mathrm{CH}$ s in their environment. We explore a new sensor node deployment and generate a stochastic geometry for the algorithm designed M-Gear which can show how the critical parameters are able to minimize energy costs based on statistics for dead nodes using the Voronoi Cell Method of stochastic optimization as shown in Fig. 6.

We developed an algorithm based on the concept suggested by Nadeem et al [28] to calculate energy across the network if the source and destination are known. We evaluate the cost and benefit of the data fusion to adaptively adjust whether fusion shall be performed to minimize total energy consumption when energy-efficient node scheduling migrates from one node to the next. One of the most important weaknesses of LEACH [29] is load

www.ausmt.org

Copyright (C) 2015 International Journal of Automation and Smart Technology unbalance, i.e., as the $\mathrm{CH}$ are selected randomly, some nodes may be selected as $\mathrm{CHs}$, which are in close proximity of each other. The range-based algorithm uses the maximum likelihood method or trilateral rule to measure the distance between nodes, and thus calculate the location of the unknown node. When the mobile node needs to be positioned, the strength values serve as an indicator of the distance between the sending and receiving motes, and show a decline in the number of beacon nodes at the path due to multipath and fading effects:

$$
\left.P_{\text {loss }}=10 \phi(h, H, d)+\omega\right)
$$

where $(h, H, d)$ reflects the relationship between the antenna height, $h$, the fracture, $H$, and the transmission distance, $d$. The parameters change with rock burst stage. Due to obstacles in a critical environment for sensor positioning measurements of the distance estimation through variance, RSSI needs to be measured from multiple reference points selected based on the power loss as shown in the M-Gear protocol whereby the network lifetime and throughput is sufficiently increased. The RSSI strength value matching location algorithm needs to create and update a large capacity signal intensity distribution for the database which entails a considerable maintenance workload. According to the RSSI algorithm, the node receives the signal strength calculated based on the signal propagation loss. From Friis' equation [30], the received signal strength (RSS) in free space at a distance, $r$ from the transmitter is expressed in logarithmic form as $P_{r}=P_{t}+G_{r}+G_{t}-L_{0}$; where $P_{t}$ is the transmit power, and ' $\mathrm{G}_{r}$ ' and $\mathrm{G}_{\mathrm{t}}$ are the gains of the receiver and transmitter antennae, respectively, while $L_{0}$ is the path loss in free space in $\mathrm{dB}$. We developed a Minimum Energy Relay Routing algorithm to determine minimum energy paths for selecting the relay node, using the dynamic path loss parameter, $L_{p}$ to minimize communication energy using the exact path loss parameter after attenuation and slip surface behavior 
analysis [31]. The beacon nodes in the UWSN relay burdensome information transmission tasks, and the lifetime of the beacon node has a great influence on the network quality of service and survival time, making it crucial to develop a UWSN routing protocol which can reduce energy consumption and extend beacon node service lifetimes. The beacon node sends a positioning request packet to its corresponding node ID, along with location information for dissemination. This is found to be highly beneficial in the re-transmission and the accurate calculation of optimum power consumption.

$$
L_{p}=6.4+20 \log (d)+20 \log (\beta)+8.69 \alpha d
$$

Equation (2) was defined in (Silva and Vuran, 2010) [32], where $d$ is the distance in kilometers between the transmitter and the receiver, $f$ is the operation frequency in $\mathrm{MHz}$, and the phase shifting constant, $\beta$ is in radian $/ \mathrm{m}$. In the free space $\lambda_{0}=c / f$, where $\beta$ is the phase shifting constant, $c=3 \times 10^{8} \mathrm{~m} / \mathrm{s}$, and $f$ is the operating frequency in $\mathrm{Hz}$. The monitoring effectiveness depends on the coverage ratio of the wireless sensor network in terms of continuous working time, data transmission time and other factors. Changes of onsite link quality can have a significant impact on ground motion prediction analysis results [33]. Site effects on attenuation relations can also have a significant impact on seismic risk. These effects are probabilistic descriptions of the level of ground motion as a function of earthquake and site parameters. Campbell's attenuation form [34] can reasonably predict the characterization of ground motion attenuation.

$$
Y_{r}(g)=f(M, R)=b_{1} e^{b_{2} M}\left(R+b_{4} \exp \left(b_{5} M\right)\right)^{-b_{3}}
$$

where, $Y_{r}$ is peak ground acceleration , $M$ is magnitude, and $R$ is source-site distance. The significant feature of the Campbell form is the exponential term which describes the magnitude dependence of the transition from nearfield to far-field attenuation and is suitable to cancel out the small changes in distance that can affect the RSSI value. This reflects a distance saturation dependant on the extent of fault rupture. Especially given the likelihood that smaller events are more prone to mis-picking of signal strengths, poor sensor azimuthal distribution and therefore mis-location, these events should be less important in the assessment of seismic activity and their contribution should be down-weighted. The denser the network and the more accurate the data, the smaller the influence of the numerical procedure. A dynamic RSSI location algorithm is proposed for a complex study set up for beacon node chain deployment in tunnel-based wireless sensor networks subject to a number of factors including interference from other objects and attenuation caused by barriers. The algorithm accounts for the distance and the corresponding RSSI between the adjacent beacon nodes to calculate the actual path loss parameter in the tunnel, thus improving the environmental adaptation of the location algorithm as shown in earlier in $[35,36]$ for mining environments. The beacon nodes are arranged in three sets in descending order of their RSSI values. The RSSI value is assumed to be greater than the other nodes and beacon nodes derive the position of the unknown node. The site-dependent ground-motion prediction models are set up and ready for use. Once the distances between the adjacent beacons nodes are known, the power output or the signal strength between the neighbor beacon nodes can be found to identify the internodal distance for a radio channel. The propagation model is obtained along with the path of each beacon node in the current context for the fading index. The relationship between the transmitting power of the wireless signal and the receiving power is usually represented by the channel transmission model shown in Eq. (4):

$$
P_{i}(d)=P_{0}+X_{0}-10 \eta \log _{10}\left[d / d_{0}\right]
$$

In the RSSI model, signal attenuation is related to the signal transmission distance. As the transmission distance decreases, the power attenuation accelerates, and vice versa.

$$
d=d_{0} \cdot 10^{\frac{P_{\text {loss }}-\omega-\psi(H)}{10 \eta}}
$$

where, $P_{i}(d)$ represents the receiving power $(\mathrm{dBm}), P_{0}$ represents the transmitting power when the reference distance is $\mathrm{d}, X_{0}$ is a Gaussian random variable with a mean of $0, d_{0}$ is the maximum node reference transmission distance, and $\eta$ is the path loss coefficient. Using this model, we can predict the optimal antenna height, increase the overall wireless network signal transmission rate, and reduce unnecessary path loss. Through the model depicted in Fig. 7, we can predict the strength of received signals and the effective signal transmission range, and reduce redundancy in the deployment of sensor nodes. In wireless communication, multipath is a signal propagation characteristic that results in a single transmission reaching a destination via two or more paths. The multipath process can originate from many sources, and is commonly caused by obstacles such as rocks reflecting the wireless signal. Some localization algorithms mitigate the multipath effects better than others.

An extreme overestimate ranging error occurs when mote ' $i$ ' receives a ranging packet from mote ' $j$ ' that is 


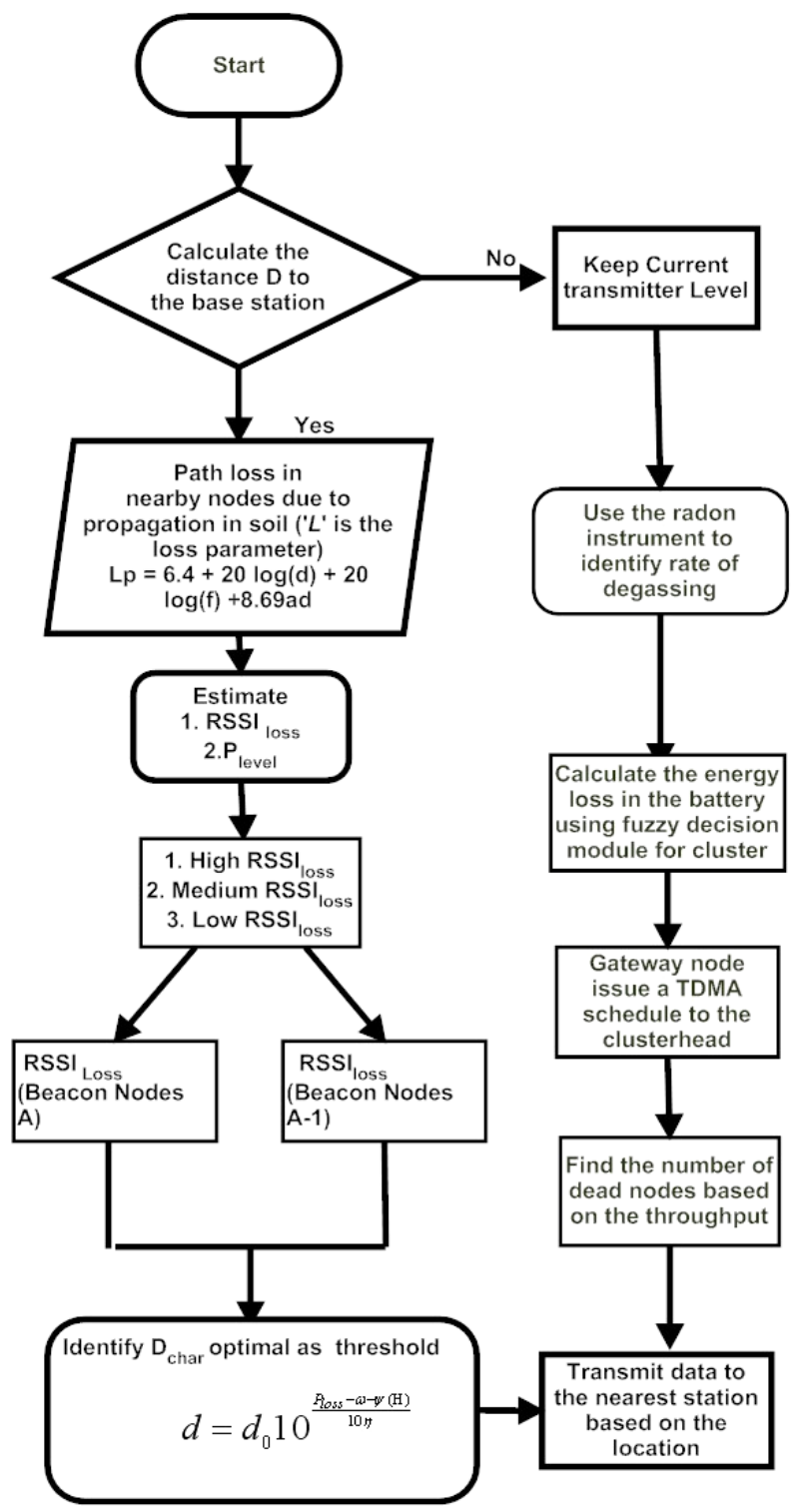

Figure 7. Flow chart of reference node in RSSI loss identification.

subject to greater signal attenuation than the true distance between the two nodes warrants. We identify the RSSI error and find that our method actually produces a smaller location analysis error, as shown in Fig. 8. Recent research has brought into question the efficacy of using RSSI as a distance estimate technique within WSNs. In an underground environment, the communication cost is high and clock synchronization across different sensor nodes is extremely difficult, thus the received signal strength method can be used to locate the target through a weighted RSSI. The RSSI is a measurement of the power level for the receiver signal. RSSI localization is a low cost technique, suitable for low-complexity networks as it does not require additional hardware if every point in the area is within range of a sensor. Specialized WSN protocols are needed to fully realize autonomous geophysical monitoring procedures using the self-potential monitoring technique.

\section{Discussion and Future perspectives}

As mentioned above, complex signal transmission environments can incur multiplat effects which reduce the strength of wireless signals. The traditional RSSI localization algorithm produces large errors and stress waves are deformed types of waves. The proposed model allows predictions of received signal strength and effective signal transmission range, thus reducing redundant deployment of sensor nodes. It also provides a guideline for quick deployment of wireless sensor network nodes while accounting for gradual changes to environmental characteristics. Using this model, we can predict the optimal antenna height, increase the overall wireless network signal transmission rate, and reduce unnecessary path loss. Data collected through such networks can be used to identify and characterize dynamic failure mechanisms of potentially hazardous rock mass failures. It can also be used to assess the usefulness of seismic monitoring as an indicator for underground tunnel design performance, and to determine empirical relationship between levels of ground shaking, seismic event magnitude, and distance to seismic sources. Several prior studies have shown that sub-surface layers beneath tectonically-complex regions are generally associated with extensive cracks and fractures, which facilitate the amount of attenuation of transmitted signals when propagated through multi-layered earth media due to variability in the velocity distribution in the sub-surface rock medium [23-25; 37-38]. The extent of high attenuation zones beneath the ocean sub-surface [39 - 40] is another cause of concern. Prevalence of structural defects in the form of fractures and cracks at varying depths may contribute significantly towards appreciable signal attenuation resulting in the reduced received signal strength recorded by underground wireless networks. The proposed algorithm may help address the issue of plausible identification of rupture locations with reduced positioning errors. The distance and the signal intensity value for the path loss in the current environment allows for the calculation of the path loss coefficient evaluation with the RSSI algorithm. This approach shows that the dynamic RSSI algorithm reduces the average error and standard deviation for positioning by $33 \%$ and $28 \%$, respectively. The dynamic algorithm outperforms the RSSI algorithm to greatly improve positioning accuracy.

Use of stronger radio transmitter beacon nodes can reduce hardware requirements along with beacon node density. The proposed method allows for the selfpositioning of beacon nodes that does not require precise positioning of the instruments in large-scale mine-fields. Our integrated model for development of earthquake early warning systems (Fig. 9) may be helpful in mitigating 


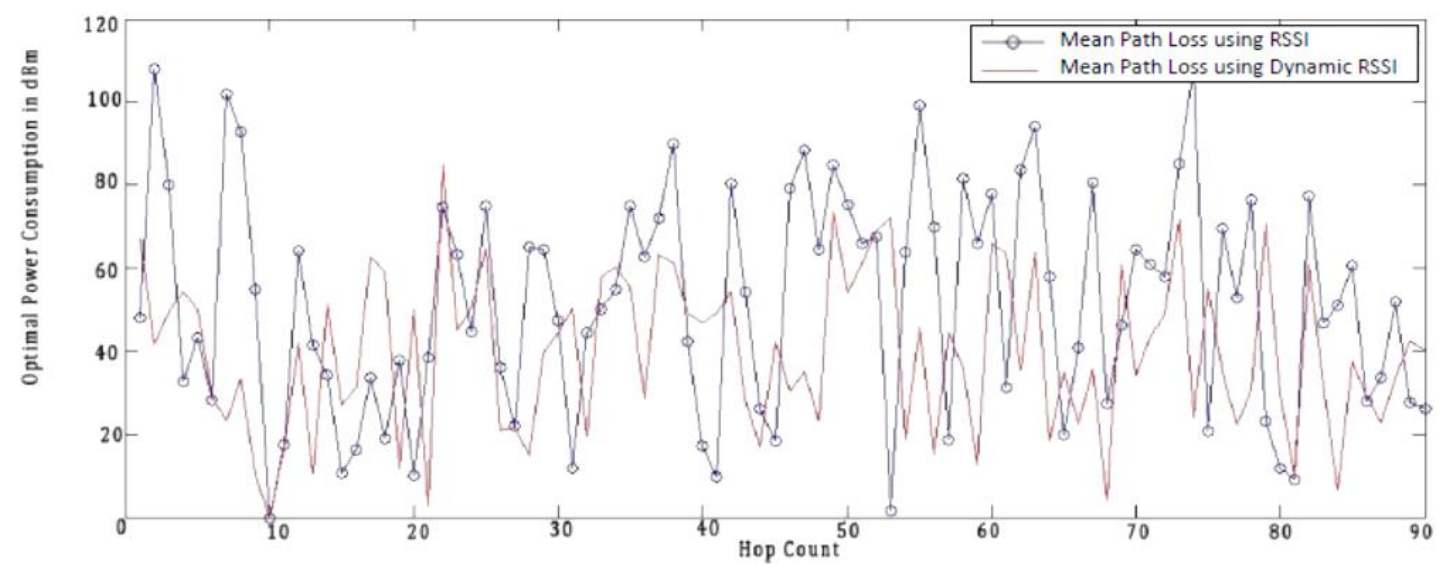

Figure 8. Node error analysis based on RSSI and dynamic path RSSI.

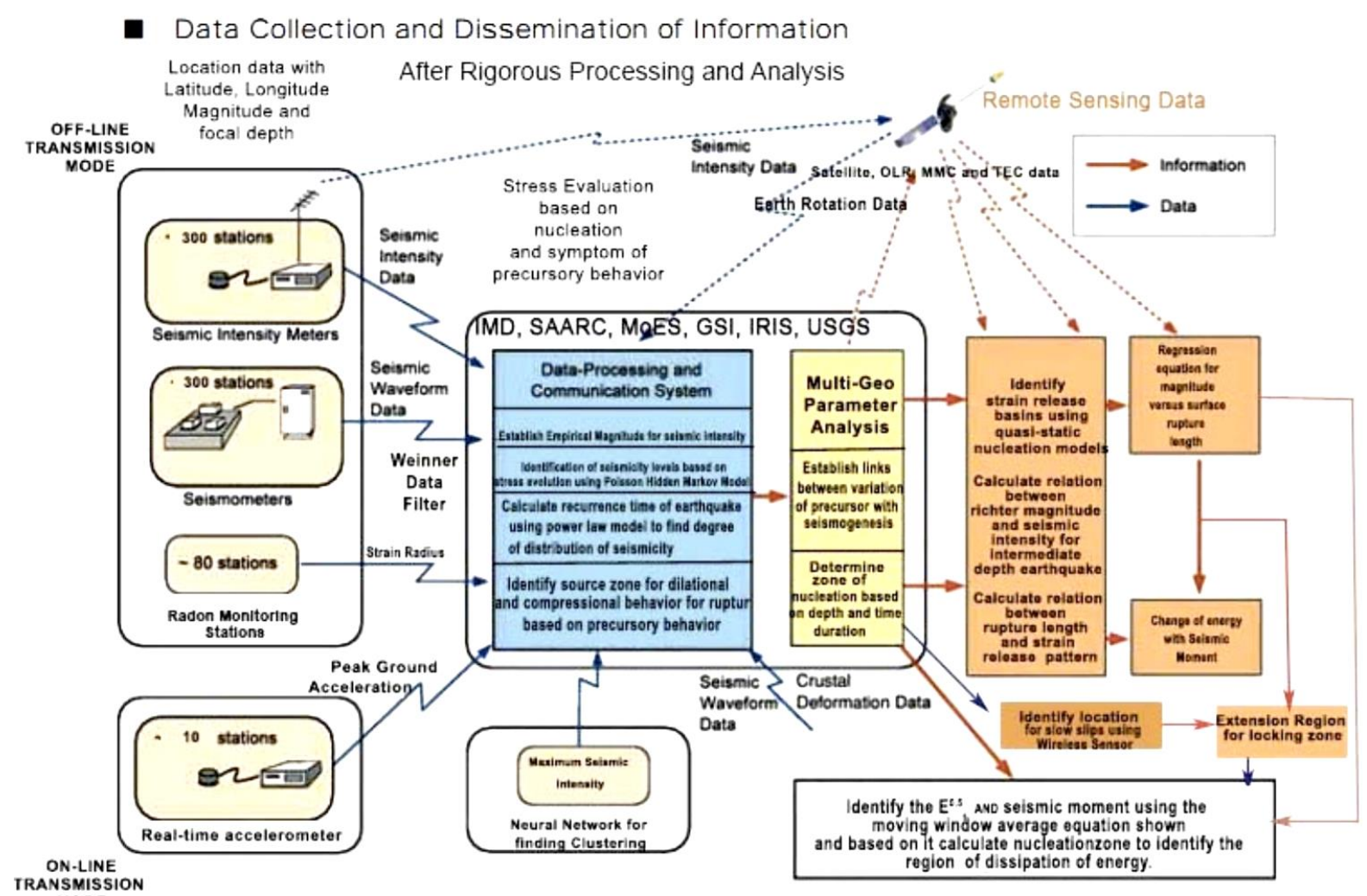

Figure 9. Integrated earthquake warning model with precursor validation.

earthquake hazards, and is in accordance with the operational earthquake forecast model for earthquake early warning systems framed by $[41,42]$ for the MARSite earthquake project for the Maramara region. Earthquake early warning systems need to meet conditions established as part of the WP4, WP6 and WP7 specifications of the REAKT project for wireless sensor design [43] to estimate the ability of forcing processes (strain variations, pore pressures, and rate of seismic energy release) and identifies a priori estimates of the "vulnerability" of the closest faults observed through spatial slip correlations, consistent with those of seismological slip maps. We recommend this model be field tested by researchers and engineers working in the fields of seismology, telecommunications, and information technology.

\section{References}

[1] D. Zhao, O. P. Mishra, and R. Sanda, "Influence of fluid and magma on earthquakes: seismological evidence," Physics of the Earth and Planetary Interiors, vol. 132, no. 4, pp. 249-267, 2002. doi: doi:10.1016/S0031-9201(02)00082-1

[2] O. P. Mishra, D. Zhao, C. Ghosh, Z. Wang, O. P. Singh, B. Ghosh, K. K. Mukherjee, D. K. Saha, G. K. Chakrabortty, and S. G. Gaonkar, "Role of crustal heterogeneity beneath Andaman - Nicobar Islands 
and its implications for coastal hazard," Natural Hazards, vol. 57, no. 1, pp. 51-64, 2011.

doi: $10.1007 / \mathrm{s} 11069-010-9678-3$

[3] O. P. Mishra, "Three-dimensional tomography of Northeast India and Indo - Burma Region and its implications for Earthquake Risks," in proceeding of National Workshop Volume on Earthquake Risk Mitigation: Strategy in North East, Guwahati, India, Feb. 24-25, 2011, pp. 40-54.

[4] O. P. Mishra, "Seismological Research in India," in proceeding of the Indian National Science Academy, vol. 78, no. 3, pp. 361-371, 2012.

[5] P. K. Dutta, M. K. Naskar, and O. P. Mishra, "Test of strain behavior model with radon anomaly in earthquake prone zones," Himalayan Geology, vol. 33, no. 1, pp. 23-28, 2012. doi: 10.4236/ijg.2012.31015

[6] M. Wyss and S. Matsumura, "Most likely locations of large earthquakes in the Kanto and Tokai areas, Japan, based on the local recurrence times," Physics of the Earth and Planetary Interiors, vol. 131, no. 2, pp. 173-184, 2002. doi: 10.1016/S0031-9201(02)00036-5

[7] O. P. Mishra, D. Zhao, N. Umino, and A. Hasegawa, "Tomography of northeast Japan forearc and its implications for interplate seismic coupling," Geophysical Research Letters, vol. 30, no. 16, pp. 71-7-4, 2003. doi: $10.1029 / 2003$ GL017736.

[8] H. Poor, An Introduction to Signal Detection and Estimation, New York: Springer-Verlag, 1985, ch. 4

[9] P. K. Dutta, "Earthquake generating mechanism and earthquake warning system design," Ph.D. dissertation, Department of Electronics and TeleCommunication Engineering, Jadavpur University, Kolkata, India, 2014.

[10] B.R. Arora, V. K. Gahalaut, and Naresh Kumar, "Structural control on along-strike variation in the seismicity of the northwest Himalaya," Journal of Asian Earth Sciences, vol. 57, pp. 15-24, 2012. doi: 10.1016/j.jseaes.2012.06.001

[11] N. Laevna, B. Elena, and K. Konstantin. "Wireless System Monitoring Seismic Activity," Ukraine Patent 102352, 2013.

[12] P. K. Dutta, O. P. Mishra, and M. K. Naskar, "Integrated process-control technology for response to disasters in operational analysis of relief scheduling," in proceeding of 9th General Assembly of Asian Seismological Commision, Mongolia, Sep. 17-20, 2012.

[13] T. Lay, Hiroo Kanamori, C. J. Ammon, K. D. Koper, A. R. Hutko, L. Ye, H. Yue, and T. M. Rushing, "Depthvarying rupture properties of subduction zone megathrust faults," Journal of Geophysical Research: Solid Earth, vol. 117, no. B4, pp. 311-332, 2012. doi: 10.1029/2011JB009133

[14] S. A. Golden and S. S. Bateman, "Sensor measurements for Wi-Fi location with emphasis on time-of-arrival ranging," IEEE Transactions on Mobile Computing, vol. 6, no. 10, pp. 1185-1198, 2007. doi: 10.1109/TMC.2007.1002

[15] C. Alippi and G. Vanini, "A RSSI-based and calibrated centralized localization technique for Wireless Sensor Networks," in proceeding of the 4th Annual IEEE International Conference on Pervasive Computing and Communications Workshops, Pisa, Italy, March 13-17, 2006 pp. 301-306. doi: 10.1109/PERCOMW.2006.13

[16] Z. Gang, T. He, S. Krishnamurthy, and J. A. Stankovic, "Impact of radio irregularity on wireless sensor networks," in proceeding of the 2nd International Conference on Mobile Systems, Applications, and Services, Boston, MA, June 6-9, 2004, pp. 125-138. doi: $10.1145 / 990064.990081$

[17] K. Whitehouse, C. Karlof, and D. Culler, "A practical evaluation of radio signal strength for rangingbased localization," ACM SIGMOBILE Mobile Computing and Communications Review, vol. 11, no. 1, pp. 41-52, 2007. doi: $10.1145 / 1234822.1234829$

[18] S. Lee and K. Kim, "Localization with a mobile beacon in underwater acoustic sensor networks," Sensors, vol. 12, no. 5, pp. 5486-5501, 2012. doi: $10.3390 / \mathrm{s} 120505486$

[19] D. Estrin, R. Govindan, J. Heidemann, and S. Kumar "Next century challenges: Scalable coordination in sensor networks," in proceeding of the 5th annual ACM/IEEE International Conference on Mobile Computing and Networking, Seattle, Washington, Aug. 15-19, 1999. pp. 263-270. doi: $10.1145 / 313451.313556$

[20] J. Tang and P. Fan, "A RSSI-based cooperative anomaly detection scheme for wireless sensor networks," in proceeding of $3^{\text {rd }}$ IEEE International Conference on Wireless Communications, Networking and Mobile Computing, New York, October 8-10, 2007, pp. 2783-2786.

doi: 10.1109/WICOM.2007.691

[21] T. He, C. Huang, B. M. Blum, J. A. Stankovic, and T. Abdelzaher, "Range-free localization schemes for large scale sensor networks," in proceeding of the 9th Annual International Conference on Mobile Computing and Networking, San Diega, California, Sep. 14-19, 2003, pp. 81-95. doi: $10.1145 / 938985.938995$

[22] D. Niculescu and B. Nath, "Error characteristics of ad hoc positioning systems (APS)," in proceeding of the 5th ACM International Symposium on Mobile Ad hoc Networking and Computing, Tokyo, Japan, May 2426, 2004, pp. 20-30. doi: $10.1145 / 989459.989463$

[23] O. P. Mishra and D. Zhao, "Crack density, saturation rate and porosity at the 2001 Bhuj, India, earthquake hypocenter: a fluid-driven earthquake," Earth and Planetary Science Letters, vol. 212, no. 3, ausMT Vol. 5 No. 3 (2015) 
pp. 393-405, 2003.

doi: $10.1016 /$ S0012-821X(03)00285-1

[24] O. P. Mishra, "Intricacies of the Himalayan seismotectonics and seismogenesis: need for integrated research," Current Science, vol. 106,no. 2, pp.176-187, 2014.

[25] O. P. Mishra, A. P. Singh, D. Kumar, and B. K. Rastogi, "An insight into crack density, saturation rate, and porosity model of the 2001 Bhuj earthquake in the stable continental region of western India," Journal of Asian Earth Sciences, vol.83, pp. 48-59, 2014. doi: 10.1016/i.jseaes.2014.01.008

[26] O. P. Mishra, P. K. Dutta, and M. K. Naskar, "Relationship between earthquake fault triggering and societal behavior using ant colony optimization," Advances in Physics Theories and Applications, vol. 26, pp. 99-108, 2013.

[27] P. K. Dutta, M. K. Naskar, and O. P. Mishra, "Impact of two-level fuzzy cluster head selection protocol for earthquake wireless sensor network: An Energy efficient approach," International Journal of Information Processing, vol. 7, no. 2, pp. 69-81, 2013. doi: arXiv: 1308.0690.

[28] Q. Nadeem, M. B. Rasheed, N. Javaid, Z. A. Khan, Y. Maqsood, and A. Din, "M-GEAR: Gateway-Based Energy-Aware Multi-Hop Routing Protocol for WSNs," in proceeding of 8th IEEE International Conference on Broadband and Wireless Computing, Communication and Applications, Compiegne, France, October 28-30, 2013, pp. 164-169. doi: 10.1109/BWCCA.2013.35

[29] G. Kumar, V. Paul, and K. P. Jacob, "Mobility metric based leach-mobile protocol," in proceeding of 16th IEEE International Conference on Advanced Computing and Communications, Chennai, India, Dec. 14-17, 2008, pp. 248-253. doi: 10.1109/ADCOM.2008.4760456

[30] L. Li, M. C. Vuran, and I. F. Akyildiz, "Characteristics of underground channel for wireless underground sensor networks," in proceeding of 6th Annual Mediterranean Ad Hoc Networking Workshop, Corfu, Greece, June 12-15, 2007, pp.92-99.

[31] E. A. Garich, "Wireless, automated monitoring for potential landslide hazards," Ph.D. dissertation, Department of Civil Engineering, Texas A\&M University, Brazos County, Texas, 2007. doi: $1969.1 / 5889$

[32] A. R. Silva and M. C. Vuran, "Development of a testbed for wireless underground sensor networks," EURASIP Journal on Wireless Communications and Networking, 2010. doi: $10.1155 / 2010 / 620307$

[33] R. E. Abercrombie and J. R. Rice, "Can observations of earthquake scaling constrain slip weakening,"
Geophysical Journal International, vol. 162, no. 2, pp.406-424, 2005.

doi: 10.1111/i.1365-246X.2005.02579.x

[34] K. Imanishi and W. L. Ellsworth, "Source scaling relationships of microearthquakes at Parkfield, CA, determined using the SAFOD pilot hole seismic array," Earthquakes: Radiated Energy and the Physics of Faulting, pp.81-90, 2006.

doi: $10.1029 / 170 \mathrm{GM} 10$

[35] K. W. Campbell, "Near-source attenuation of peak horizontal acceleration," Bulletin of the Seismological Society of America, vol. 71, no. 6, pp. 2039-2070, 1981.

[36] Z. J. Chen, S. J. Jiang, and X. J. Chen, "Research on routing of WSN in mine," Application Research of Computers, vol. 25, no. 12,pp. 3747-3749, 2008.

[37] S. Padhy, O. P. Mishra, D. Zhao, and W. Wei, "Crustal heterogeneity in the 2007 Noto-Hanto earthquake area and its geodynamical implications," Tectonophysics, vol. 509, no. 1, pp. 55-68, 2011. doi: 10.1016/i.tecto.2011.06.002

[38] A. P. Singh, O. P. Mishra, R. B. S. Yadav, and D. Kumar, "A new insight into crustal heterogeneity beneath the 2001 Bhuj earthquake region of Northwest India and its implications for rupture initiations," Journal of Asian Earth Sciences, vol.48, pp. 31-42, 2012. doi: 10.1016/i.jseaes.2011.12.020

[39] O. P. Mishra, D. Zhao, and D. D. Singh, "Northwest Pacific fundamental mode Rayleigh-wave group velocity and its relationship with tectonic structures," Bulletin of the Seismological Society of America, vol. 95, no. 6, pp.2125-2135, 2005. doi: $10.1785 / 0120030201$

[40] O. P. Mishra, D. Zhao, and D. D. Singh, "Surface wave studies beneath the Pacific Ocean," Bulletin of the Seismological Society of America, vol. 95, no. 6, pp.2152-2161, 2005. doi: $10.1785 / 0120030134$

[41] S. Xiao, X. Wei, and Y. Wang, "A multipath routing protocol for wireless sensor network for mine security monitoring," Mining Science and Technology (China), vol. 20, no. 1, pp. 148-151, 2010. doi: 10.1016/S1674-5264(09)60177-0

[42] P. Gasparini and G. Manfredi, Early Warning for Geological Disasters, Heidelberg, Germany: Springer, 2014. doi: $10.1007 / 978-3-642-12233-05$

[43] M. Erdik, Y. Fahjan, O. Ozel, H. Alcik, A. Mert, and M. Gul, "Istanbul earthquake rapid response and the early warning system," Bulletin of Earthquake Engineering, vol. 1, no. 1, pp. 157-163, 2003. doi: $10.1023 / \mathrm{A}: 1024813612271$ 